\title{
HEPATECTOMY USING MICROWAVE TISSUE COAGULATOR IN LIVER CANCER
}

\section{ASSOCIATED WITH CIRRHOSIS}

Katsuyoshi Tabuse, M. D., Yoji Tabuse, M. D., Yasuhito Kobayashi, M. D.,

Kazunari Mori, M. D., Seiki Yamamoto, M. D., Masahiro Sakaguchi, M. D.,

Nakahiro Shimotsuma, M. D., Koji Minami, M. D., and Miho Nagahama, M. D.

\begin{abstract}
We have performed hepatectomy using microwave tissue coagulator which we have developed in about 122 patients with liver cancer associated with cirrhosis. Merit of this high hemostatic performance has been put to use from the first and operations with this have been considered to be those which hardly require transfusion during operation. We introduce you the keys and points to be regarded of recent operative technique. Points in operation can be summarized as follows : 1. Portal blocking is not made, 2. Cholecystectomy is made and a catheter is placed in the cystic duct, 3 . The line for incision is determined previously by ultrasonography, 4. Coagulation is made mimutely, strongly and in no hurry ( $80 \mathrm{~W}$. 45s), 5 . A line for incision is determined to leave coagulation zone which extends from the electrode impaling site to $10 \mathrm{~mm}$ thereof sufficiently on the side of the remaining liver. 6. Coagulation in the neighborhood of large vessels is avoided, 7. Even though cutting suture occurred at the ligature of small vessel systems, Z-type ligature is applied afterward, 8 . When the resection is finished the absence of stump bleeding is confirmed and then indigocamine is infused from a tube which is placed previously and biliary leakage test is made without fail, 9. Omentopexy is made without fail to remove a dead space between the subdiaphragmatic surface and liver. 10. The drain is removed as early as possible to prevent retrograde infection.
\end{abstract}

Dept. of Surgery, Osaka-Minami National Hospital

Address : 677-2, Kido-Cho, Kawachinagano, Osaka, Japan.

Telephone : 0721-53-5761 Telex : 0721-53-8904

Key Words : Hepatectomy, Microwave Tissue Coagulator, Hepatocellural Carcinoma and Liver cirrhosis. 


\section{Introduction}

Technique of hepatectomy and therapeutic results of liver cancer with cirrhosis has made a rapid progress recently. Since 1978, when the first clinical application wa done in hepatoma rapture case by the microwave tissue coagulator which I have devised.

Hepatectomies using this apparatus were undergone in about 300 cases for a variety of conditions.

The characteristics of this device is following 3 points (1) in this method, which utilizes microwave of $2,450 \mathrm{MHz}$ in feqnences and $12 \mathrm{~cm}$ in wave length, thermal energy radiating from the monopolar electrode can be focalized to target tissue, (2) its properties of tissue coagulation and hemostasis are excellent and (3) the device is easily and safely operable.

The operations have become stable ones owing to improvement of resection technique, devices during operation, improvement of postoperative regimen and so forth. In this paper, we introduce the key and points to be regarded of recent operative technique with a microwave tissue coagulator.

\section{OUTLINE OF THE DEVICE AND OPERATIVE MANIPULATION}

This device, Microtaze ${ }^{\circledR}$ model HSD-20M, manufactured by Heiwa Electronic Industries, Ltd., and distributed by Nippon Koden, Ltd., is composed of a main body, having self-contained microwave generator, cavity magnetron connector, various safety devices and tissue dissociation apparatus, a coaxial cable, and an operable electrode.

When a needle electrode is inserted into a tissue, and microwave are emitted through the electrode, the tissue around the electrode, articularly around the base of the electrode, is strongly coagulated. At the time of withdrawal of the electrode, if the tissue dissociation apparatus is operated through manipulation of the foot switch, the electrode can be smoothly drawn off without adhesion of the coagulated tissue because water is produced between the tissue and electrode as a result of electrochemical reaction. Although differing among tissue, coagulation is conducted at 60-80 watts with radiation lasting 30-60 seconds at a time, and followed by manipulation of tissus dissociation for 10-20 seconds. In liver with cirrhosis, the standard output and irradiation time employed by our department are 80 watts and 60 seconds, respectively. Tissue dissociation becomes more effective as taking more time. 


\section{CASE}

Patient : 63 years old, male.

Chief complaint : right hypochondrial pain.

History : thoracoplasty.

Family history : father, esophageal cancer; elder brother, lung cancer; elder sister, lung cancer; younger brother, leukemia.

Course of the present disease : This patient visited a nearby physician because of hypochondrial pain and anorexia, and he was diagnosed as having liver cancer based on ultrasonographic findings and a high AFP level. After transarterial embolization (TAE) was performed 3 times, he was referred to our department.

On postoperative examination, his Alb was $3.7 \mathrm{~g} / \mathrm{dl}$, the total Bilirubin was $0.3 \mathrm{mg} / \mathrm{dl}$, the hepaplastin test gave $68 \%$, the ICGR15 was $12.6 \%$, and the ICGK was 0.139 .

Operative procedure : We selected the transphrenic approach through right thoracotomy and laparotomy. As CT scans show, his tumor occupied $\mathrm{S}_{7}$ and $\mathrm{S}_{8}$. Although the tumor diminished in size after TAE, it still received the blood supply from the inferior phrenic artery. Therefore, the right lobe of liver was exposed while a portion of the diaphragm was simultaneously resected. Resection was thought to be limited to segmentectomy of $S_{7-8}$ because the liver was found to be complicated with cirrhosis of type $Z$, and a range of resection was decided through intraoperative ultrasography. As shown is Fig. 1A, the liver tissue was punctured with a needle electrode, microwave knife, while the middle hepatic vein was preserved. Prior to the initial puncture, a depth (a length of the needle electrode inserted) and an angle were decided ultrasonography. After making a decision, the portal vein system. It is recommended that portal branches feeding a tumor are ligated and selected as soon as possible during the process of liver resection. As shown in Fig. 1B, coagulation should be performed as carefully as possible so as to coagulate the tissue in the from of belt at small intervals as possible, and the tissus around the tumor should be made ischemic (dark black).

Because the resection surface in this patient was too wide to be made ischemic by one layer of punctures using a needle electrode of $3 \mathrm{~cm}$ length, punctures were repeated for $2 \mathrm{nd}$ and 3rd layers. Although such repeated procedure of coagulation and resection seems to be timeconsuming and cumbersome, and would make us undue hasty, bleeding may occur and result in taking more time at the time of ligation and resection if puncture is cased before achievement of complete coagulation. It is important to reach feeding portal branches as possible, and these vein 
are then ligated and resected (Fig. 2). At the same time, small hepatic veins should be ligated and resected as many as possible. Intrahepatic vessels should be carefully ligated using 3-0 suture to avoid any cutting sutures. If a cutting occurs, bleeding should be certainly stopped by letter-of- $z$ suture using $5-0$ or $3-0$ Nespiren ${ }^{\circledR}$. As a result, the resected surface is entirely coagulated, and bloodless resection is accomplished. In this patient, the right common hepatic vein and about 4 short hepatic veins were ligated and resected at their junctions to the inferior vena cava at the time of which the liver was turned over and taken out.

In Fig. 3, bile leakage from the resected surface is being examined. Namely, the pigment indigocarmine (about $15-20 \mathrm{ml}$ ) is injected through a nodulated catheter inserted into the cystic duct in advance following cholecystectomy while the end of the common bile duct is closed with compression by finger, and then leakage of the pigment from the resected surface is observed. If any site becomes stained, such site is ligated with letter-of- $Z$ suture using $5-0$ Nespiren ${ }^{\circledR}$. Through sound accomplishment of this procedure, we can completely eliminate bile leaks from the resected surface. Although there is practically no bleeding or bile leakage from the resected surface of the liver, Abiten ${ }^{\circledR}$ is applied to the hepatic parenchyma at the insertion of the inferior vena cava, on which we cannot utilize hemostatic coagulation.

Before the beginning of closure of the abdomen after irrigation of the abdominal cavity, the greater omentum is treated (namely, resection of the pedicle), and sutured together with the resected liver surface. It is especially important to eliminate any dead spaces between the liver and diaphragm for management of postoperative drainage. Other than this, a length of time for retaining a drain at the edge of the resected liver has been limited to 1 week on average, or 2

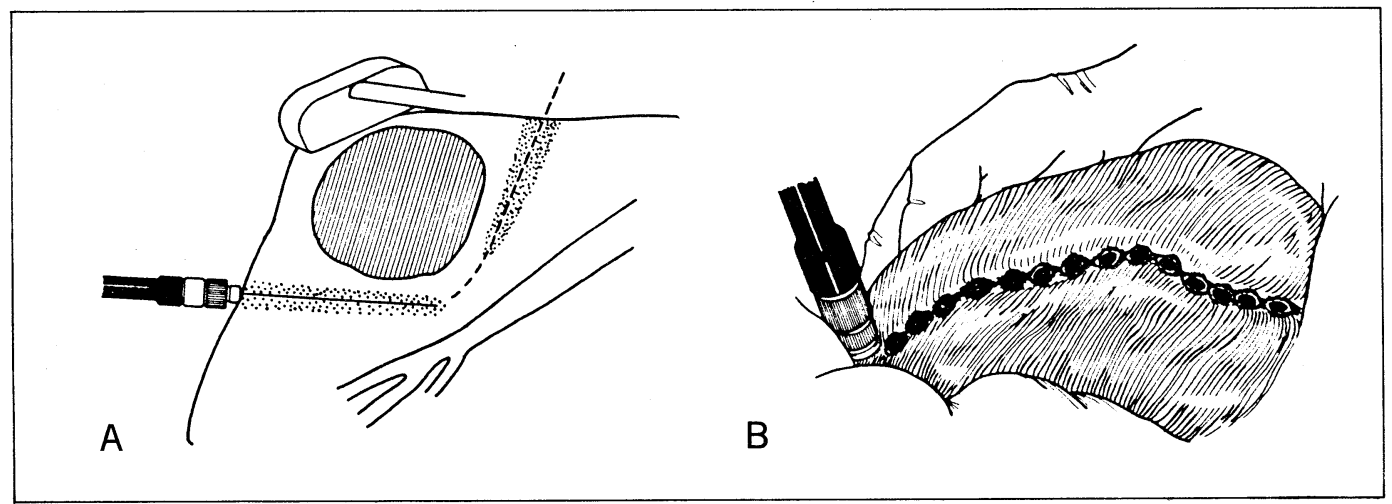

Fig. 1A. The needle electrode is inserted under the guide of US to preserve the middle hepatic vein.

(1) Middle hepatic vein

Fig. 1B. Coagulation should be carefully performed at small intervals as possible.

(1) Tumor 


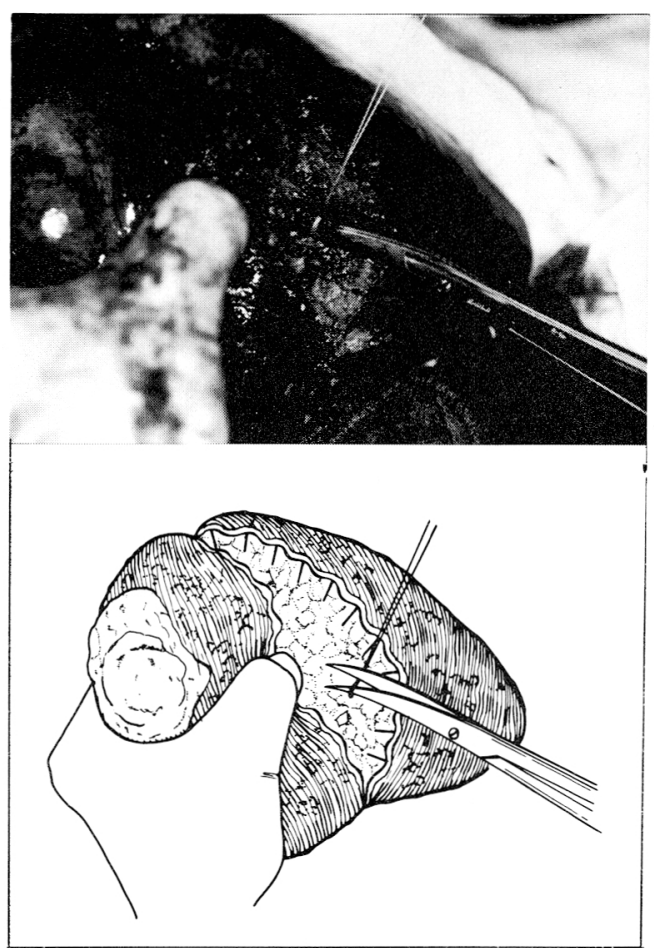

Fig. 2. Feeding portal branches should be ligated and resected as early as possible.

(1) Feeding portal branch
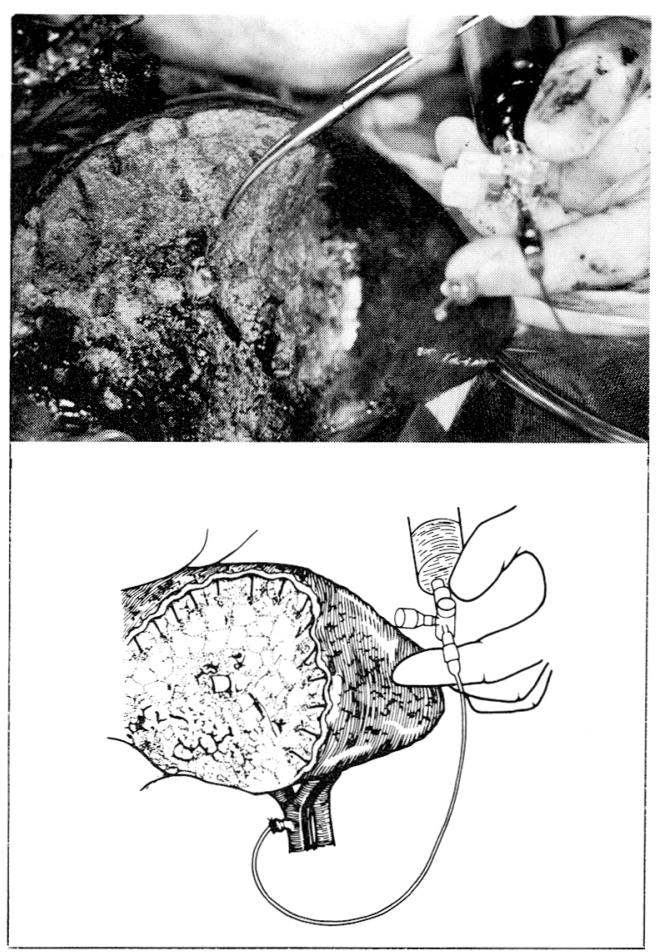

Fig. 3. Injection of the pigment indigocarmine (bile leak test).

weeks in the longest cases. Conversely, it is important not to retain a drain for a long period of time, because incidence of retrograde infection through the drain and resulting abscess formation could minimized through short-term retention.

This patient had retention of pleural effusion as a complication, but showed no subphrenic abscess formation. At the present time, 3 years after operation, he is in good condition, showing no evidence of recurrence.

\section{IMPORTANT POINTS IN OPERATION}

Besed on the results obtained from the patient described here, important points in operation are summarized below :

(1) Strong coagulation is secured by punctures performed at small intervals without being in a hurry.

(2) The liver should be resected along a line up to $10 \mathrm{~mm}$ apart from the line of needle electrode punctures in order to leave a sufficient amount of completely coagulated layer on the 
remaining part of the liver.

(3) We should avoid coagulating a tissue in the vicinity of a large blood vessel.

(4) If a cutting suture occurs, it should be ligated later with letter-of- $Z$ suture.

(5) It is especially important to perform the bile leak test, in which indigo carmine is injected into the cystic duct.

(6) Dead spaces made by suture of the greater omentun should be eliminated.

(7) A drain should be removed as early as possible to prevent the occurrence of retrograde infection.

\section{MERITS AND DEMERITS}

\section{Merits}

(1) This technique provides most superior hemostasis, and makes, therefore, bloodless operation possible.

(2) Blockade of the portal vein is unnecessary.

(3) Operation can be achived without destruction of the hilar area.

(4) TW can be sufficiently maintained in the cases of liver cancer.

(5) It can be also used for the reduction of tumor volume as a coagulation therapy.

(6) The coagulated layer is healed without sloughing.

(7) It is the best method of liver resection in the presence of cirrhosis.

(8) It is very useful for atypical hepatectomy.

(9) Its operative manipulation is highly safe.

\section{Demerits}

(1) Tissue is considerably damaged by heat.

(2) A coagulated and necrotized layer requires a long time for absorption.

(3) It is safe in manipulation, but requires a long time for both tissue coagulation and dissociation.

(5) Main vessels should be preserved from thermal damages. 


\section{Summary}

Since we developed a microwave tissue coagulator (Microtaze ${ }^{\circledR}$ ), not pnly an electric knife, but also laser knife, an ultrasonic surgical aspirator (CUSA), cryosurgery, and water jet have been improved and remodeled as surgeons have undertaken coagulation, hemostasis and destruction of parenchymatous organs, mainly for liver resection, using these devices each in its proper way with careful consideration of its merits and demerits. Although intraoperative bleeding has been the greatest problem in liver resection, this problem can be almost completely solved by applying our microwave coagulation method, which reduces an amount of intraoperative bleeding to less than $1,000 \mathrm{ml}$ and provides a bloodless surgical field. It has been also confirmed that a resected liver surface can be healed without having postoperative complications of liver resection if the liver resected by the microwave coagulation method along a line leaving a completely coagulated layer on the remaining part of the liver. As for demerits, it has been criticized that coagulation takes considerably time, particularly when an area requiring coagulation is wide. Although there may be many alternatives of solving this problem, we have continuously studier the point a tissue based on the thought that parallel running of microwave generators is the best way at the present time.

\section{References}

(1) Tabuse K. A new operative procedure of hepatic surgery using a microware tissue coagulator. Arch Jpn Chir 1979, 48 : 160-172.

(2) Tabuse K and Katsumi M. Application of a Microware Tissue Coagulator to Hepatic Surgery-The Hemostatic Effects on Spontaneous Rupture of Hepatoma and Tumor Necrosis-. Arch Jpn Chir 1981,50 : 571-579.

(3) Tabuse K, Katsumi M. Kobayashi Y. et al. Microwave Surgery : Hepatectomy Using a Microwave Tissue Coagulator. World J Surg 1985,9 : 136-143. 